Maria Augusta Aliperti Ferreira ${ }^{1}$

MILENA LAVOR ${ }^{1}$

Gullherme Machado de Carvalho'

Alexandre Caixeta Guimarães'

Vanessa Gonçalves Siliva ${ }^{1}$

JorGe RizZATo PAsChOAL ${ }^{2}$

Artigo Original

Palavras-chave

Complicações na gravidez

Paralisia facial

Paralisia de Bell

Período pós-parto

Corticosteroides

Keywords

Pregnancy complications Facial paralysis

Bell palsy

Postpartum period

Adrenal cortex hormones

Puerperium

\title{
Paralisia facial periférica e gestação: abordagem e tratamento
}

\author{
Facial palsy and pregnancy: management and treatment
}

\section{Resumo}

OBJETIVO: Comparar o grau da paralisia facial periférica de gestantes e puérperas no momento da admissão e na alta e avaliar outros fatores associados. MÉTODOS: Estudo retrospectivo, transversal, com análise dos prontuários de gestantes e puérperas atendidas no ambulatório de paralisia facial, em um período de 12 meses, com aplicação de protocolo padronizado de avaliação das pacientes e da escala de House-Brackmann na primeira consulta e na data da alta. RESULTADOS: Foram identificadas 6 pacientes, com média de idade de 22,6 anos. Cinco casos foram classificados com estadiamento IV e um com II na escala de House-Brackmann, sendo que duas eram puérperas e quatro gestantes. Todas evoluíram com melhora na escala de House-Brackmann. CONCLUSÃO: A paralisia de Bell tem bom prognóstico mesmo em gestantes e puérperas, sendo importante realizar tratamento adequado para diminuir as sequelas neste grupo apontado como mais susceptível à paralisia facial periférica.

\section{Abstract}

PURPOSE: To compare the degree of peripheral facial palsy of pregnant women and puerperae at admission and at discharge and to evaluate related factors. METHODS: Retrospective, cross-sectional study, with analysis of medical records of pregnant and postpartum women with facial palsy, over a period of 12 months, with application of a standardized protocol for patient evaluation and of the House-Brackmann scale on the occasion of the first visit and at discharge. RESULTS: Six patients were identified, mean age of 22.6 years. Five cases were classified as stage IV and one as stage II on the House-Brackmann scale, being two of them puerperae and four pregnant. All showed improvement on the House-Brackmann scale. CONCLUSION: The Bell's palsy has a good prognosis even in pregnant and postpartum women, being important to perform the correct treatment to reduce the sequelae in this group identified as more susceptible to peripheral facial palsy.

Correspondêncio

Maria Augusta Aliperti Ferreira Rua Tessália Vieira de Carvalho, 126 CEP: $13083-887$ Campinas (SP), Brasil

Recebido 19/04/2013
Trabalho realizado no Hospital das Clínicas da Universidade Estadual de Campinas - UNICAMP - Campinas (SP), Brasil. 'Serviço de Otorrinolaringologista, Universidade Estadual de Campinas - UNICAMP - Campinas (SP), Brasil.

${ }^{2}$ Serviço de Base de Crânio, Disciplina de Otorrinolaringologia, Cabeça e Pescoço, Universidade Estadual de Campinas - UNICAMP - Campinas (SP), Brasil.

Conflito de interesses: não há 


\section{Introdução}

A paralisia facial periférica idiopática (PFP) é uma disfunção do nervo facial que acomete indivíduos de todas as idades, podendo causar alterações emocionais, sociais e profissionais. A paralisia aguda do nervo facial é uma entidade clínica comum com a qual todo otorrinolaringologista deve estar familiarizado ${ }^{1}$.

A paralisia de Bell (PB) é a causa mais comum de PFP aguda unilateral, com incidência estimada de 24 a 40 acometidos a cada 100.000 pessoas $^{2-4}$. Há relato de incidência de 20,2/100.000,0 pessoas por ano na população em geral, sendo menor a encontrada em menores de 14 anos e maior o número de casos novos verificados na população geriátrica, com 75 anos ou mais ${ }^{5}$.

Trata-se de uma paralisia periférica aguda do nervo facial de causa não definida, sendo o diagnóstico de exclusão $0^{3,6}$. Acredita-se em alguns possíveis agentes causais, como infecção viral, doença vascular, hipertensão e diabetes, mas a reativação do herpes simplex tipo 1 parece ser a principal causa ${ }^{4}$. Em 57 a $70 \%$ dos acometidos é registrada recuperação completa da paralisia sem tratamento medicamentoso ${ }^{2,7}$, e $30 \%$ dos pacientes persistem com algum grau de paralisia e/ou sequela ${ }^{7}$.

Sir Charles Bell foi o primeiro a utilizar o termo paralisia facial idiopática e a descrever sua associação com a gestação, em $1830^{2,8}$. A taxa de prevalência de PB na gestação é estimada em 45,1 casos para cada 100.000,0 mulheres, que é consideravelmente maior que da população feminina em idade fértil não gestante $^{9}$. Em revisão sistemática, observou-se que a maioria dos casos de PB foi constatada no terceiro trimestre de gestação ou no puerpério imediato ${ }^{10,11}$.

A taxa de hipertensão gestacional e pré-eclâmpsia é maior nas gestantes com PB comparando à população obstétrica em geral ${ }^{12,13}$. O prognóstico de recuperação considerado satisfatório é significativamente pior em mulheres que desenvolvem PFP de Bell durante a gravidez quando levada em conta a população em geral $^{13}$, o que torna fundamental o conhecimento de ginecologistas e obstetras sobre a PFP, sua evolução e suas complicações incomuns ${ }^{13}$.

A PFP idiopática é a complicação dos pares cranianos mais frequente da gravidez. Apesar da PFP ser uma complicação neurológica frequente na gestação, esta afecção ainda permanece incomum no período puerperal tardio ${ }^{14,15}$. Não há evidência conclusiva de que a etiologia da PB em gestantes seja diferente da de mulheres não gestantes. A alteração de susceptibilidade à reativação do herpes simples durante a gestação é a explicação mais aceita para a maior concentração de casos no terceiro trimestre ${ }^{16}$.

A avaliação clínica do grau de paralisia facial é um dado subjetivo e que difere de examinador para examinador. Diversos sistemas têm sido propostos para a padronização de uma escala universal, sendo o Sistema de House-Brackmann o mais amplamente aceito e adotado pela Academia Americana de Otorrinolaringologia. Tendo em vista o possível risco que a gestação e o puerpério representam para a ocorrência de PFP, e seu prognóstico menos favorável, este estudo tem o objetivo de comparar o grau da paralisia facial de gestantes e puérperas atendidas em serviço médico especializado no momento da admissão com o momento da alta, e avaliar outros fatores associados, além de realizar uma revisão da literatura.

\section{Métodos}

O presente estudo é retrospectivo, transversal e realizado com informações obtidas por meio da análise dos prontuários de gestantes e puérperas atendidas no ambulatório de paralisia facial da disciplina de Otorrinolaringologia, Cabeça e Pescoço do Hospital de Clínicas da Universidade Estadual de Campinas até agosto de 2012. Foi utilizado um protocolo padronizado de avaliação das pacientes e feita a comparação do grau de paralisia facial de acordo com a escala de House-Brackmann ${ }^{17,18}$ (Quadro 1) na primeira consulta e na data da alta.

Foram incluídos apenas prontuários de pacientes gestantes ou puérperas (até oito semanas após o parto) com $\mathrm{PB}$, atendidas nos últimos 12 meses, que apresentavam todas as informações requisitadas pelo protocolo de coleta de dados, sendo os casos incompletos excluídos.

No protocolo de coleta de dados de nosso serviço constam os seguintes itens: idade da paciente; idade gestacional ou tempo de puerpério; lado da PFP com graduação baseada na escala de House-Brackmann; avaliação audiológica com audiometria e pesquisa de reflexo estapediano; otoscopia; presença ou ausência de sintomas associados (hiperacusia, otalgia, parestesia,

Quadro 1. Escala de House-Brackmann ${ }^{17,18}$

\begin{tabular}{|c|c|c|}
\hline Grau & Função & Descriç̣̃ó \\
\hline I & Normal & Função normal em todas as áreas \\
\hline II & Disfunção leve & Leve fraqueza na inspeção cuidadosa \\
\hline III & Disfunção moderada & Óbvio, mas não desfigurante \\
\hline IV & Disfunção moderada-grave & Fraqueza óbvia e/ou assimetria desfigurante \\
\hline V & Disfunção grave & Movimento pouco perceptível \\
\hline VI & Paralisia total & Sem movimento \\
\hline
\end{tabular}


alteração do paladar, sincinesias); comorbidades; tipo do parto; avaliação; e documentação fotográfica com fonoaudióloga.

Para o tratamento das pacientes utilizamos como protocolo o corticoide sistêmico na dose de $1 \mathrm{mg} / \mathrm{kg}$ por 7 dias, com esquema de retirada até completar 15 dias. Houve cuidados oculares com oclusão noturna do olho afetado com pomada oftalmológica durante a noite e uso de lágrima artificial durante o dia. Consideramos alta para as pacientes com melhora na escala de House-Brackmann (menor que III), sem repercussões funcionais.

Considerando a revisão narrativa da literatura, foram usados os seguintes descritores: "paralisia facial periférica e gestantes", "paralisia facial periférica e puérperas", "paralisia facial periférica e tratamento" e "paralisia facial periférica e complicações". Foram incluídos artigos do PubMed/MedLine e Google Scholar de língua inglesa e portuguesa dos últimos 15 anos e respeitados os preceitos do comitê de ética da instituição local.

\section{Resultados}

Identificaram-se seis pacientes com média de idade de 22,6 anos. Em cinco casos, elas tiveram estadiamento na escala de HB IV, e em um, HB II, sendo o lado direito acometido em quatro casos. Duas pacientes eram puérperas e o restante, gestante. $\mathrm{O}$ tempo de seguimento das pacientes entre a data da primeira consulta até a melhora da paralisia $(\mathrm{HB} \leq \mathrm{III})$ variou de 7 a 30 dias, e todas as pacientes tiveram melhora na escala de House-Brackmann. Elas receberam tratamento com corticoide sistêmico. Nas gestantes, a PFP apareceu entre a $24^{\mathrm{a}}$ e a $36^{\mathrm{a}}$ semana de gestação, e nas puérperas variou do $3^{\circ}$ ao $28^{\circ}$ dia de puerpério. Em relação às comorbidades, duas pacientes apresentavam hipertensão arterial sistêmica gestacional; uma diabetes gestacional, hipotireoidismo e hiperprolactinemia; uma diabetes mellitus insulino-dependente; e duas negaram comorbidades (Tabela 1 ).

\section{Discussão}

Vários mecanismos potenciais foram propostos para explicar a patogênese da PB em gestantes. Alterações durante a gravidez têm sido postuladas para contribuir ou causar o aparecimento da PFP aguda, incluindo um estado de hipercoagubilidade, hipertensão ou eclâmpsia, aumento da água corporal total, mudanças nos níveis de progesterona e estrogênio, crescimento do nível de cortisol, imunossupressão e aumento da susceptibilidade às infecções virais, particularmente

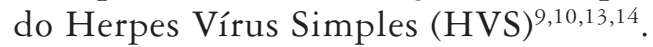

A maioria dos casos de PFP durante a gestação (aproximadamente $71 \%$ ) ocorre no terceiro trimestre gestacional e no puerpério imediato, caracterizado pelos 10 primeiros dias após o parto (aproximadamente $21 \%$ ), com a maioria deles na proximidade do parto ${ }^{15}$.

Diversas hipóteses foram aventadas como mecanismos etiológicos desta complicação no período puerperal, incluindo trauma cirúrgico, acidentes vasculares, causas infecciosas, características hereditárias e mecanismos autoimunes ${ }^{15}$. Foi observada associação entre a PFP e as doenças hipertensivas específicas da gestação, com prevalência da paralisia em aproximadamente $29 \%$ das pacientes com pré-eclâmpsia, comparando com 5\% das gestantes sem este distúrbio ${ }^{14,15,19}$.

Acredita-se que a hipertensão arterial observada nestas pacientes leva ao aumento dos fluidos extravasculares, resultando em edema perineural e compressão do nervo facial no canal de falópio. A ocorrência da pré-eclâmpsia como fator de risco para a PFP foi observada não somente durante a gestação, mas também em pacientes com pré-eclâmpsia leve que apresentaram a sintomatologia no puerpério imediato ${ }^{15,20}$.

Entretanto, a explicação mais provável é a reativação da infecção latente do HVS dentro do gânglio geniculado ${ }^{21,22}$. Para reforçar esta evidência, em 1996, Murakami et al. ${ }^{22}$ identificaram o DNA do HVS em $79 \%$ das mulheres grávidas com $\mathrm{PFP}^{21}$. Além disso, a PB é mais comum no terceiro trimestre da gestação ou no puerpério imediato (até dez dias de pós-parto),

Tabela 1. Características epidemiológicas e clínicas de seis pacientes com paralisia facial periférica

\begin{tabular}{|c|c|c|c|c|c|c|}
\hline & Idade (anos) & Lado & Início da PFP & Sintomas associados & Grau inicial & Grau na alta \\
\hline Caso 1 & 20 & D & $3^{0}$ dia puerperal & Nenhum & IV & $\|$ \\
\hline Caso 2 & 21 & $E$ & $28^{\circ}$ dia puerperal & Parestesia & $\|$ & I \\
\hline Caso 4 & 20 & D & $36^{0}$ semana & Alteração no paladar & IV & $\|$ \\
\hline Caso 5 & 26 & $E$ & $29^{a}$ semana & Hiperacusia & IV & I \\
\hline
\end{tabular}

PFP: paralisia facial periférica; D: direito; E: esquerdo. 
quando é observada reativação maior também do HVS, na sua apresentação de vesículas orais ${ }^{21,23}$.

Uma pesquisa com mulheres no início da gravidez (10 a 15 semanas de gestação) mostrou que a frequência da reativação do HSV oral foi reduzida nas que tinham história de herpes oral ${ }^{24}$. Assim, nas primeiras semanas de gestação ocorre uma supressão na reativação do HSV, explicando potencialmente a relativa escassez de casos de paralisia de Bell no primeiro e segundo trimestres. Também existe a teoria de que a reativação do HSV oral no período pós-parto imediato pode ser aumentada pela utilização de morfina epidural ou intratecal, permitindo a especulação de que alguns casos de Bell pós-parto possam ser consequência da anestesia de parto ${ }^{25}$.

Embora a PB seja a etiologia mais frequente de PFP em grávidas, é essencial excluir outras causas, como herpes zoster, neoplasia, otite média aguda, colesteatoma, malformação artério-venosa, trauma, miastenia gravis e caxumba ${ }^{9}$. A varicela zoster vírus foi também implicada na patogênese da PFP, mesmo sem erupções da pele ("zoster sineherpete") ${ }^{26,27}$.

A maioria das gestantes com PB apresenta fraqueza facial unilateral aguda (24 a 48 horas), que pode progredir para paralisia facial completa ou quase completa dentro de uma semana. Frequentemente existem sintomas associados, incluindo dor, febre, olho seco, alterações do paladar e salivação e hiperacusia ${ }^{11}$.

O tratamento típico da PB inclui corticosteroides orais, antivirais, cuidados tópicos com os olhos e descompressão cirúrgica do nervo ${ }^{5,6}$. Entretanto, algumas vezes este tratamento não é prescrito em casos de mulheres grávidas devido aos potenciais riscos para a mãe e o feto. Os riscos maternos incluem: exacerbação de úlceras pépticas, psicose, retenção de fluidos e exacerbação do diabetes e da osteoporose. Já entre os riscos fetais estão a supressão da adrenal, o baixo peso ao nascer e malformações como fissura palatina, quando o tratamento acontece no primeiro trimestre ${ }^{28}$.

Sendo assim, o tratamento da PFP em gestantes com prednisona permanece controverso também devido à possibilidade da piora da imunossupressão fisiológica da gestação, podendo agravar o curso natural da doença ${ }^{28}$. Apesar do benefício do uso deste medicamento na gravidez não ter sido totalmente comprovado, é comum durante a gestação devido ao baixo risco apresentado à mãe e ao feto ${ }^{6}$. Os medicamentos antivirais representam outro tratamento adjuvante para a paralisia de Bell. Análogos de nucleosídeos, incluindo o aciclovir, valaciclovir e famciclovir, são classificados na categoria B na gravidez e representam pouco risco à mãe ou ao feto ${ }^{29}$. Estes agentes são geralmente empregados para a prevenção de surtos de HSV no final da gravidez ${ }^{30}$.

Dois trabalhos duplo-cegos randomizados controlados demonstraram melhores resultados em pacientes com PB tratados com esteroides e medicamentos antivirais em comparação com esteroides por si só ş, $^{3,31}$. O benefício potencial provavelmente supera o risco de usar estes medicamentos, embora, para se ter eficácia máxima nos antivirais, eles devam ser iniciados nos três dias do quadro de paralisia facial ${ }^{5}$.

Entretanto, observou-se que a recuperação da PFP em pacientes gestantes se mostrou mais rápida do que em não gestantes, independente do tratamento farmacológico. A presença de esteroides endógenos durante a gestação, assim como a faixa etária das gestantes acometidas pela paralisia, é fator que pode explicar este fenômeno ${ }^{32}$.

Os cuidados oculares em pacientes com PFP representam a parte menos controversa do tratamento, devendo ser os mesmos em pacientes gestantes e não gestantes. Os olhos precisam ser lubrificados durante o dia com colírios tipo lágrima artificial para a proteção da córnea contra abrasões ${ }^{6}$ e, durante a noite, é recomendado o uso de pomadas e tampões oclusivos, além da colocação de umidificadores nos quartos ${ }^{3,6}$. As complicações oculares do tratamento inadequado incluem desde o lacrimejamento excessivo ate a úlcera de córnea com perda da visão.

O prognóstico para pacientes grávidas com PFP unilateral incompleta é excelente, com recuperação quase total ou sequelas mínimas na maioria dos $\operatorname{casos}^{33}$. O prognóstico é reservado para aquelas que progridem com a paralisia completa, com déficits persistentes em até $50 \%$ dos casos. Porém, a possibilidade de uma forma mais grave de PFP em grávidas é suportada pelos achados de Peitersen ${ }^{2}$, que afirma que as grávidas não se recuperam bem quando o tratamento não é administrado. Hilsinger et al. reforçam este dado, demonstrando ausência de diferença na recuperação de mulheres grávidas e não grávidas, quando todas foram submetidas ao tratamento com esteroides ${ }^{2,9,11}$. Sendo assim, as evidências que sugerem que o prognóstico é pior em pacientes grávidas, podem refletir um viés histórico do tratamento inadequado delas ${ }^{14}$.

A PFP é uma situação que merece atenção especializada, principalmente em grupos específicos, como nos casos relacionados à gestação e ao puerpério.

Houve melhora do grau da paralisia periférica em todas as pacientes. Deve-se atentar para instituir um tratamento adequado, melhorando o prognóstico e a qualidade de vida delas. 
1. Baricich A, Cabrio C, Paggio R, Cisari C, Aluffi P. Peripheral facial nerve palsy: how effective is rehabilitation? Otol Neurotol. 2012;33(7): $1118-26$.

2. Peitersen E. Bell's palsy: the spontaneous course of 2,500 peripheral facial nerve palsies of different etiologies. Acta Otolaryngol Suppl. 2002;(549):4-30.

3. de Almeida JR, Al Khabori M, Guyatt GH, Witterick IJ, Lin VY, Nedzelski JM, et al. Combined corticosteroid and antiviral treatment for Bell palsy: a systematic review and meta-analysis. JAMA. 2009;302(9):985-93.

4. Monini S, Lazzarino Al, lacolucci C, Buffoni A, Barbara M. Epidemiology of Bell's palsy in an Italian Health District: incidence and case-control study. Acta Otorhinolaryngol ltal. 2010;30(4):198.

5. Rowlands S, Hooper R, Hughes R, Burney P. The epidemiology and treatment of Bell's palsy in the UK. Eur J Neurol. 2002;9(1):63-7.

6. Axelsson $S$, Berg $T$, Jonsson L, Engström M, Kanerva M, Pitkäranta $A$, et al. Prednisolone in Bell's palsy related to treatment start and age. Otol Neurotol. $2011 ; 32(1): 141-6$.

7. Hydén D, Sandstedt $P$, Odkvist LM. Prognosis in Bell's palsy based on symptoms, signs and laboratory data. Acta Otolaryngol. 1982;93/5 6):407-14.

8. Klein A. Peripheral nerve disease in pregnancy. Clin Obstet Gynecol. 2013;56(2):382-8.

9. Hilsinger RL Jr, Adour KK, Doty HE. Idiopathic facial paralysis, pregnancy, and the menstrual cycle. Ann Otol Rhinol Laryngol. 1975;8414 Pt 1):433-42.

10. Shapiro JL, Yudin MH, Ray JG. Bell's palsy and tinnitus during pregnancy: predictors of pre-eclampsia? Three cases and a detailed review of the literature. Acta Otolaryngol. 1999;1 19(6):647-51.

11. Gillman GS, Schaitkin BM, May M, Klein SR. Bell's palsy in pregnancy: a study of recovery outcomes. Otolaryngol Head Neck Surg. 2002;126(1):26-30.

12. Sibai BM, Caritis SN, Thom E, Klebanoff M, McNellis D, Rocco L, et al. Prevention of preeclampsia with low-dose aspirin in healthy, nulliparous pregnant women. The National Institute of Child Health and Human Development Network of Maternal-Fetal Medicine Units. N Engl J Med. 1993;329(17):1213-8.

13. Ragupathy K, Emovon E. Bell's palsy in pregnancy. Arch Gynecol Obstet. 2013;287(1):177-8.

14. Ushio M, Kondo K, Takeuchi N, Tojima H, Yamaguchi T, Kaga $\mathrm{K}$. Prediction of the prognosis of Bell's palsy using multivariate analyses. Otol Neurotol. 2008;29(1):69-72.

15. Shmorgun D, Chan WS, Ray JG. Association between Bell's palsy in pregnancy and pre-eclampsia. QJM. 2002;95(6):359-62.

16. Mylonas I, Kästner R, Sattler C, Kainer F, Friese K. Idiopathic facial paralysis (Bell's palsy) in the immediate puerperium in a patient with mild preeclampsia: a case report. Arch Gynecol Obstet. 2005;272(3):241-3.

17. Fawale MB, Owolabi $M O$, Ogunbode O. Bell's palsy in pregnancy and the puerperium: a report of five cases. Afr J Med Med Sci. 2010;39(2):147-51.
18. House JW, Brackmann DE. Facial nerve grading system. Otolaryngol Head Neck Surg. 1985;93(2): 146-7.

19. Vrabec JT, Backous DD, Dialilian HR, Gidley PW, Leonetti JP, Marzo SJ, et al. Facial nerve grading system 2.0. Otolaryngol Head Neck Surg. 2009; 140(4):445-50.

20. Fujiwara T, Hato N, Gyo K, Yanagihara N. Prognostic factors of Bell's palsy: prospective patient collected observational study. Journal Eur Arch Otorhinolaryngol. 2013 Sep 1. [Epub ahead of print]

21. Mathieu N, Ledigabel JF. Pre-eclampsia and facial paralysis. Gynecol Obstet Fertil. $2011 ; 39(2):$ e31-3.

22. Murakami S, Mizobuchi M, Nakashiro Y, Doi T, Hato N, Yanagihara N. Bell palsy and herpes simplex virus: identification of viral DNA in endoneurial fluid and muscle. Ann Intern Med. 1996; 124(1 Pt 1):27-30.

23. Davies PW, Vallejo MC, Shannon KT, Amortegui AJ, Ramanathan S. Oral herpes simplex reactivation after intrathecal morphine: a prospective randomized trial in an obstetric population. Anesth Analg. 2005;100(5):1472-6.

24. Massey EW, Stolp KA. Peripheral neuropathy in pregnancy. Phys Med Rehabil Clin N Am. 2008;19(1):149-62.

25. Scott D, Moore S, Ide M, Coward P, Baylis R, Borkowska E. Recrudescent herpes labialis during and prior to early pregnancy. Int J Gynaecol Obstet. 2003;80(3):263-9

26. Dorsey DL, Camann WR. Obstetric anesthesia in patients with idiopathic facial paralysis (Bell's palsy): a 10-year survey. Anesth Analg. 1993;77(1):81-3.

27. Cohen Y, Lavie O, Granovsky-Grisaru S, Aboulafia Y, Diamant YZ. Bell palsy complicating pregnancy: a review. Obstet Gynecol Surv. 2000;55(3): 184-8.

28. Adour KK. Otological complications of herpes zoster. Ann Neurol. 1994;35 Suppl:S62-4.

29. Park-Wyllie L, Mazzotta P, Pastuszak A, Moretti ME, Beique $L$, Hunnisett $L$, et al. Birth defects after maternal exposure to corticosteroids: prospective cohort study and meta-analysis of epidemiological studies. Teratology. 2000;62(6):385-92.

30. Hato N, Yamada H, Kohno H, Matsumoto S, Honda N, Gyo K, et al. Valacyclovir and prednisolone treatment for Bell's palsy: a multicenter, randomized, placebo-controlled study. Otol Neurotol. 2007;28(3):408-13.

31. Hollier LM, Wendel GD. Third trimester antiviral prophylaxis for preventing maternal genital herpes simplex virus (HSV) recurrences and neonatal infection. Cochrane Database Syst Rev. 2008;(1):CD004946.

32. Adour KK, Ruboyianes JM, Von Doersten PG, Byl FM, Trent CS, Quesenberry CP Jr, et al. Bell's palsy treatment with acyclovir and prednisone compared with prednisone alone: a doubleblind, randomized, controlled trial. Ann Otol Rhinol Laryngol. 1996; 105(5):371-8.

33. Vrabec JT, Isaacson B, Van Hook JW. Bell's palsy and pregnancy. Otolaryngol Head Neck Surg. 2007;137(6):858-61. 